\title{
Memberships and CM diagrams of the open cluster NGC $7243^{\star}$
}

\author{
E. G. Jilinski ${ }^{1,2,3}$, V. N. Frolov ${ }^{1}$, J. K. Ananjevskaja ${ }^{1}$, J. Straume ${ }^{4}$, and N. A. Drake ${ }^{2,5}$ \\ 1 Main Astronomical Observatory, Pulkovo, St. Petersburg, Russia \\ 2 Observatório Nacional/MCT, Rua Gal. Jose Cristino 77, São Cristovão, Rio de Janeiro, Brazil \\ 3 Laboratório Nacional de Computação Científica/MCT, Petropolis, Brazil \\ ${ }^{4}$ Radioastrophysical Observatory, Baldone, Riga, Latvia \\ 5 Sobolev Astronomical Institute of St. Petersburg State University, St. Petersburg, Russia
}

Received 16 November 1999 / Accepted 6 January 2003

\begin{abstract}
The results of astrometric and photometric investigations of the open cluster NGC 7243 are presented. Proper motions

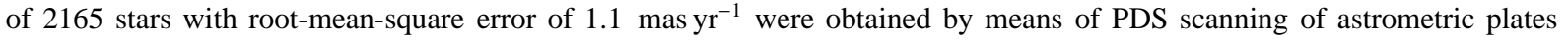
covering 97 years. A total of 211 cluster members down to $V=15.5$ mag have been identified. $V$ and $B$ magnitudes have been determined for 2118 and 2110 stars respectively. Estimations of mass $\left(348 M_{\odot} \leq M_{\mathrm{TOT}} \leq 522 M_{\odot}\right)$, age $\left(t=2.5 \times 10^{8} \mathrm{yr}\right)$, distance $(r=698 \mathrm{pc})$ and reddening $\left(E_{B-V}=0.24\right)$ of the cluster NGC 7243 have been made.
\end{abstract}

Key words. open clusters and associations: general - open clusters and associations: individual: NGC 7243

\section{Introduction}

The open cluster NGC 7243 is located in the Lacerta constellation. It has the following coordinates: $\ell=98^{\circ} .9, b=-5^{\circ} .6$, $\alpha=22^{\mathrm{h}} 15^{\mathrm{m}}, \delta=+49^{\circ} 53^{\prime}(2000.0)$. In the Lyngå (1987) catalogue it is classified as Trümpler (1930) class II $2 \mathrm{~m}$ with 40 cluster members and an angular diameter of $21^{\prime}$. Early investigations of this cluster led Raab (1922) to the conclusion that it consisted of two clusters, but a lack of sufficient observational data made it impossible to check this statement at that time. Zug (1933) was the first investigator to study the interstellar absorption in the cluster area. He denoted the brightest stars and these numbers are still used in some papers. Relative proper motions of 814 stars in the cluster area were for the first time obtained by Lengauer (1937) from plates taken with the Normal Astrograph of the Pulkovo Observatory with a 33 year mean epoch difference. As a result, 32 cluster members were selected. Later, Rahmatov \& Muminov (1985) determined proper motions of 1287 stars in the region of NGC 7243 using the plates of the Normal Astrograph in Tashkent (Uzbekistan) as the first epoch plates. Due to intensive urban light pollution, the second epoch plates had to be taken with the $40 \mathrm{~cm}$ Zeiss Astrograph of the Kitab Latitude Station (Uzbekistan). In the circle of $40^{\prime}$ radius, 28 possible cluster members with a membership probability of more than $50 \%$ were selected. van Schewick (1957), using only one pair of plates, confirmed the existence of cluster members previously found by Lengauer

Send offprint requests to: E. G. Jilinski, e-mail: jilinski@on. br

* Table 8 is only available in electronic form at the CDS via anonymous ftp to cdsarc.u-strasbg.fr $(130.79 .128 .5)$ or http://cdsweb.u-strasbg.fr/cgi-bin/qcat?]/A+A/401/531 but did not add any new ones. Unfortunately, none of the above investigations, for one reason or another (absence of photometric data, small epoch difference, small number of plate pairs, etc.) permitted investigators to select all probable members of the cluster NGC 7243 in the observed magnitude range and to estimate membership probabilities.

The Main Astronomical Observatory at Pulkovo has 19 first epoch plates (obtained from year 1897 to 1949). This allows one to solve the astrometric part of the problem with a high degree of confidence. For a reliable segregation of the cluster members it is necessary to use photometric data based on the international photometric system.

Photometric plates of the NGC 7243 region in $B$ and $V$ passbands were obtained with the Schmidt Telescope (80/120/240) of the Latvian Radioastrophysical Observatory.

\section{Observations}

The observational material used for the determination of the relative proper motions in the field of NGC 7243 is shown in Table 1. All plates are from the Normal Astrograph Collection of Pulkovo Observatory. The Pulkovo Normal Astrograph has a diameter of $33 \mathrm{~cm}$, and a scale of $60^{\prime \prime} \mathrm{mm}^{-1}$. All plates are in the $m_{\mathrm{pg}}$ passband and have been obtained with exposure times listed in Table 1. The plate and film material used for the $B V$ photometry is shown in Table 2.

\section{Astrometry}

The deepest plate (K 11) was measured by means of the semiautomatic coordinate measuring machine "Askorecord" with a 
Table 1. Astrometric plate material.

\begin{tabular}{|c|c|c|c|}
\hline Plate & $\begin{array}{c}\text { Exposure } \\
\text { (min) }\end{array}$ & Epoch & "Quality \\
\hline \multicolumn{4}{|c|}{ Early epoch } \\
\hline A 202 & 20 & 1897 Aug. 13 & poor \\
\hline A 346 & 30 & 1899 Sep. 5 & good \\
\hline A 525 & 62 & 1901 Sep. 14 & good \\
\hline A 708 & 60 & 1903 Sep. 24 & poor \\
\hline A 976 & 22 & 1907 Sep. 11 & high \\
\hline B 261 & 30 & 1910 Oct. 11 & good \\
\hline C 995 & 30 & 1933 Nov. 23 & high \\
\hline C 996 & 40 & 1933 Nov. 23 & high \\
\hline C 997 & 30 & 1933 Nov. 23 & high \\
\hline K 11 & 80 & 1934 Feb. 9 & high \\
\hline D 232 & 21 & 1949 Oct. 30 & good \\
\hline \multicolumn{4}{|c|}{ Recent epoch } \\
\hline 15453 & 10 & 1987 Oct. 18 & good \\
\hline 15454 & 10 & 1987 Oct. 18 & good \\
\hline 17670 & 10 & 1995 Oct. 15 & high \\
\hline 17671 & 20 & 1995 Oct. 15 & high \\
\hline 17696 & 10 & 1996 Jan. 24 & good \\
\hline 17697 & 10 & 1996 Jan. 24 & high \\
\hline 17704 & 10 & 1996 Jan. 25 & poor \\
\hline
\end{tabular}

precision of about $0.01 \mathrm{~mm}$. Serial numbers from 1 to 2623 were assigned to all stars in a square size of $80^{\prime} \times 80^{\prime}$ centered on the cluster. These positions were used as preliminary ones when all plates were scanned with the Observatório Nacional (Brazil) PDS 1010 microdensitometer. The stellar images were analyzed with a $10 \times 10 \mu \mathrm{m}$ diaphragm. The plates were scanned in two orientations with a 180 degree rotation to eliminate possible errors of the image treatment and to minimize the influence of systematic errors of the PDS microdensitometer. The analysis and comparison of the direct and reverse measurements permitted us to evaluate the random and systematic errors of measurements as $\pm 1.5 \mu \mathrm{m}$ in both coordinates.

The star image positions were determined through twodimensional elliptical Gaussian fits to the matrix of pixels of the image. These calculated positions of the stars do not depend on the orientation of the scanning.

The method used for proper motion determination traditionally suggests compiling of plate pairs. The combinations of plates that form such pairs are listed in Table 3 .

The mean epoch difference is 74 years. Some of the second epoch plates were used in the pairs twice. This was caused by unfavorable conditions of observation at Pulkovo Observatory due to urban lights. The plate 17704 was excluded because of poor quality of measurements. Nine plates of the first epoch were not deep enough and were not used.

The positions of stars on all plates were related to one conventional center (star 898 in our catalogue). The plates of the second epoch were used as a comparison, therefore the coordinate axes on the plates were rotated so that the axis $Y$ was oriented along the sky meridian. For this purpose stars from the catalogue AGK 3 were used.

The reference stars were chosen in the $B$ magnitude interval between $13.5 \mathrm{mag}$ and $14.5 \mathrm{mag}$. To avoid the so-called "cosmic" error, i.e. the position change of a system of reference stars because of their real movements, the following operation was applied. All the plates used were transferred on one reference plate D 232, as its epoch is the median of the others. Then for each reference star candidate, graphs were considered where on abscissa the epoch of plates and on ordinate the coordinate $X$ or $Y$ of the star were plotted. The straight line was drawn by least square fitting. The star was included in the reference stars list only if this straight line was parallel to the coordinate axis, which testified to the absence of an appreciable proper motion.

Thus, 99 reference stars (of average $B$ magnitude equal to 14.04) existing on all plates and uniformly distributed in the studied region, except the cluster area itself, were selected.

Quadratic polynomial approximations were used to match the position of the star on the the 1st epoch plate to that of the 2nd epoch:

$$
\begin{aligned}
& a x+b y+c x y+d x^{2}+e y^{2}+f=\Delta x \\
& a^{\prime} x+b^{\prime} y+c^{\prime} x y+d^{\prime} x^{2}+e^{\prime} y^{2}+f^{\prime}=\Delta y .
\end{aligned}
$$

The mean errors of star relative proper motions for each plate pair are given in Table 4.

In calculating average values of $\mu_{x}$ and $\mu_{y}$, weights of individual plate pairs were not taken into account since the difference between the mean and weighted mean do not exceed the random errors. Values of the estimated root-mean-square (rms) errors of the obtained proper motion do not depend on the position of a star in the studied field, but increase 1.5 times from bright to faint stars. The mean rms errors of the proper motion components for all observed magnitudes are the following:

$\sigma_{x}= \pm 1.09$ mas yr$^{-1}, \sigma_{y}= \pm 0.96$ mas yr$^{-1}$.

Relative proper motions were determined for 2165 stars in the investigated region.

\section{Photometry}

Photographic $B V$ photometry of the stars in the cluster area was made using the material described in Sect. 2. The measurements of the plates were performed by means of the microphotometer "Sartorius" with an iris diaphragm. The fluctuations of the zero-point of the scale of the device during the measurements were checked and introduced into instrumental magnitudes as correspondent corrections. The measured area is characterized by the existence of a large number of weak stars forming a rather irregular background. For this reason, more than $10 \%$ of all stars of the catalogue are either blended or fall simultaneously in the field of view of the device. Also, in the diaphragm, together with a measured star, one or several weak stars often enter that are not numbered. Nevertheless, the measurements of such stars were done. Stellar magnitudes obtained in this way are approximate as their errors are as high as $0.5 \mathrm{mag}$. In the final catalogue they are denoted by an attribute “:”. Other doubtful cases, for example, the absence of a star on one of the plates are marked by colon.

Usually in measurements with iris-photometers, the background of a plate is considered as regular and is not especially taken into account. However, for the field of NGC 7243 it is not 
Table 2. Photometrical plate material.

\begin{tabular}{ccccc}
\hline \hline Plate & Epoch & Passband & Photomaterial & Quality \\
\hline 18575 & 1991 Jan. 13/14 & $V$ & A600 H + YG 17 & high \\
19026 & 1992 Jan. 25/26 & $V$ & A600 H + YG 17 & high \\
19027 & 1992 Jan. 25/26 & $V$ & A600 H + YG 17 & high \\
19202 & 1992 Aug. 27/28 & $V$ & A600 H + YG 17 & high \\
18536 & 1990 Dec. 16/17 & $B$ & ZU 21 + GG 13 & high \\
19028 & 1992 Jan. 25/26 & $B$ & ZU 21 + GG 13 & good \\
19230 & 1992 Dec. 17/18 & $B$ & ZU 21 + GG 13 & high \\
\hline
\end{tabular}

Table 3. Plate pairs used for the proper motions determination.

\begin{tabular}{cccccc}
\hline \hline Pair & Plates & $\begin{array}{c}\text { Epoch } \\
\text { difference } \\
\text { yr }\end{array}$ & Pair & Plates & $\begin{array}{c}\text { Epoch } \\
\text { difference } \\
\text { yr }\end{array}$ \\
\hline 1 & A 346-17696 & 97 & 6 & C 995-17671 & 62 \\
2 & A 525-17697 & 95 & 7 & C 996-17670 & 62 \\
3 & A 708-15453 & 84 & 8 & C 997-17671 & 62 \\
4 & A 976-15670 & 88 & 9 & K 11-17696 & 62 \\
5 & B 256-15454 & 77 & 10 & D 232-17697 & 47 \\
\hline
\end{tabular}

Table 4. Errors of stars relative proper motions obtained using different plate pairs.

\begin{tabular}{cccc}
\hline \hline Plate pair & $\begin{array}{c}\text { Number } \\
\text { of stars }\end{array}$ & \multicolumn{2}{c}{$\begin{array}{c}\epsilon_{x} \\
\left(\mathrm{mas}_{\mathrm{yr}} \mathrm{y}^{-1}\right)\end{array}$} \\
\hline 1 & 2025 & 3.37 & 2.73 \\
2 & 1923 & 3.05 & 2.62 \\
3 & 2121 & 2.77 & 2.34 \\
4 & 2140 & 2.42 & 2.08 \\
5 & 2122 & 2.94 & 2.65 \\
6 & 2026 & 3.37 & 3.25 \\
7 & 2084 & 3.15 & 2.86 \\
8 & 2107 & 3.30 & 2.92 \\
9 & 1956 & 3.99 & 3.29 \\
10 & 1716 & 4.33 & 4.13 \\
\hline
\end{tabular}

so, mainly in the neighbourhood of the bright stars which, at first sight, make up the cluster. Thus, each plate was measured twice: with consideration of the background and without it. The comparison of the results showed that consideration of the background improves a convergence of magnitude values obtained with different plates by not more than 0.02 mag, considerably less than the photometric error $(0.05 \mathrm{mag})$. However, in the final catalogue the photometry obtained accounting for the background is given using the well-known formula of Weaver (1962)

$d^{2}=D^{2}-\left(d_{\mathrm{f}}^{2}-d_{\mathrm{sf}}^{2}\right)$,

where $D$ - aperture reading at prompting on a star,

$d_{\mathrm{f}}$ - aperture reading for an average background near the star,

$d_{\text {sf }}$ - aperture reading for an average standard background,

$d$ - aperture reading for a star, related to an average standard background. The background near each star was measured two to four times with subsequent averaging.

The photometric calibration of each photometric plate was done using photoelectrically measured $B$ and $V$ magnitudes of stars in the region of NGC 7243, published in two works: Mianes \& Daguillon (1956) and Hill \& Barnes (1971) (hereafter referred to as MD and HB respectively). In the first work the number of stars is 39 , in the second -20 , with 9 common stars. Since there is a systematic difference between these two sets of standards, the work of MD was used as the principal and the values $B$ and $V$ from the work of HB were reduced to its system. Three stars from the GSC (Lasker et al. 1988) were added to this list. The full list of the photoelectric standards is given in Table 5.

About 30 stars spreading regularly over the whole range of magnitudes were used to plot characteristic curves. Unfortunately, the number of photoelectric standards for faint stars is extremely low, which almost doubles the error. The characteristic curves for each photometric plate were fitted by polynomials. As an example of the calibration, a characteristic curve for plate 19026 is presented in Fig. 1.

Instrumental magnitudes were transformed into $B$ and $V$ taking into account the colour equation (CE): $V$ magnitude varies by $0.05 \mathrm{mag}$, while $B-V$ changes from -0.20 to 2.11 ; for the $B$ magnitude this variation reaches $0.15 \mathrm{mag}$. These CEs were used for every $B$ or $V$ plate transformation. The resulting $B$ and $V$ magnitudes are free of CE effects.

The external rms errors of our photometry calculated relative to the corresponding photoelectric values for 39 stars are the following:

$\sigma_{V}= \pm 0.05 \mathrm{mag}, \quad \sigma_{B-V}= \pm 0.07 \mathrm{mag}$

The internal rms errors were calculated only for stars with reliable photometry. Blended stars or stars contacted with not denoted ones, as well as stars that had not been measured on all plates obtained in the same passband were excluded. Finally, photometric magnitudes $B$ and $V$ were obtained for 2110 and 2118 stars respectively. Photometry errors increase slightly from the center of the plate to its edges. The following 
Table 5. Photoelectric photometric standard stars in the region of NGC 7243.

\begin{tabular}{rcccccrccccc}
\hline \hline $\begin{array}{r}\text { This } \\
\text { paper }\end{array}$ & Lengauer & $V$ & $B-V$ & $U-B$ & Notes & $\begin{array}{r}\text { This } \\
\text { paper }\end{array}$ & Lengauer & $V$ & $B-V$ & $U-B$ & Notes \\
\hline 912 & 475 & 8.43 & 0.11 & -0.20 & & 869 & 382 & 11.35 & 0.64 & 0.16 & $*$ \\
909 & 480 & 8.74 & 0.12 & -0.22 & $\mathrm{P}$ & 892 & 489 & 11.44 & 1.44 & 1.38 & \\
757 & 482 & 9.07 & 0.14 & -0.23 & & 743 & 476 & 11.55 & 0.33 & 0.20 & \\
879 & 377 & 9.21 & 0.08 & -0.30 & $*$ & 761 & 485 & 11.68 & 0.29 & 0.22 & \\
1085 & 380 & 9.22 & 0.09 & -0.22 & & 775 & 497 & 11.22 & 0.27 & 0.19 & \\
895 & 362 & 9.25 & 0.15 & -0.23 & $*$ & 1086 & 379 & 11.74 & 0.25 & 0.19 & \\
902 & 358 & 9.35 & 0.13 & -0.21 & $\mathrm{Q}$ & 1097 & 384 & 11.79 & 0.26 & 0.18 & \\
896 & 361 & 9.60 & 0.12 & -0.26 & $*$ & 897 & 363 & 11.79 & 0.26 & 0.16 & \\
1078 & 370 & 9.93 & 0.05 & -0.26 & & 881 & 372 & 11.80 & 0.23 & 0.18 & \\
771 & 490 & 10.10 & 0.12 & -0.26 & & 762 & 488 & 11.82 & 0.62 & 0.18 & $\mathrm{~T}$ \\
898 & 360 & 10.27 & 1.75 & 2.10 & & 1068 & 364 & 11.93 & 0.39 & 0.23 & \\
1099 & 386 & 10.27 & 0.15 & -0.06 & $\mathrm{R} *$ & 1084 & 378 & 12.08 & 0.27 & 0.20 & $*$ \\
776 & 496 & 10.39 & 0.13 & -0.08 & & 781 & 502 & 12.19 & 0.42 & 0.30 & \\
908 & 350 & 10.40 & 0.45 & 0.20 & & 907 & 355 & 12.20 & 0.72 & 0.29 & \\
880 & 373 & 10.50 & 0.62 & 0.16 & $*$ & 778 & 501 & 12.21 & 0.34 & 0.24 & \\
876 & 510 & 10.54 & 0.19 & 0.00 & & 922 & 338 & 12.29 & 0.40 & 0.28 & \\
1081 & 374 & 10.66 & 0.72 & 0.27 & & 888 & 366 & 12.30 & 0.45 & 0.36 & \\
768 & 493 & 10.73 & 0.21 & -0.05 & & 1090 & 376 & 12.37 & 0.44 & 0.28 & $*$ \\
924 & 471 & 10.76 & 0.20 & -0.01 & & 905 & 483 & 12.41 & 0.48 & 0.29 & \\
1104 & 392 & 10.86 & 0.13 & 0.00 & & 875 & 511 & 12.42 & 0.37 & 0.26 & \\
1075 & 368 & 10.89 & 0.14 & -0.01 & & 877 & 375 & 12.50 & 0.46 & 0.26 & \\
874 & 513 & 11.08 & 0.70 & 0.25 & $\mathrm{~S} *$ & 772 & 494 & 12.66 & 0.41 & 0.28 & $\mathrm{G}$ \\
759 & 484 & 11.15 & 0.22 & 0.05 & & 792 & 515 & 12.69 & 0.42 & & \\
904 & 487 & 11.20 & 0.18 & 0.01 & $*$ & 793 & & 12.77 & 1.29 & & $\mathrm{H}$ \\
780 & 503 & 11.24 & 0.29 & 0.21 & $*$ & 884 & 505 & 12.83 & 0.25 & -0.02 & \\
886 & 369 & 11.34 & 0.19 & 0.11 & $*$ & 918 & & 15.04 & 0.73 & & $\mathrm{I}$ \\
\hline
\end{tabular}

1) * - The star merging in blend or close contact with weak star(s) without number.

2) By capital letters are denoted the stars of the photometric sequence of the catalogue GSPS (plate P-188).

3) For the star 886 in the catalogue of MD faulty photometry is given.

4) The star 872 (512) is not included in the list, since its $V$ magnitude determined by us is one magnitude brighter than that given in MD (MD: $V=12.62 \mathrm{mag}, B-V=0.35 \mathrm{mag}$; the authors catalogue: $V=11.52 \mathrm{mag}, B-V=0.24 \mathrm{mag}$ ).

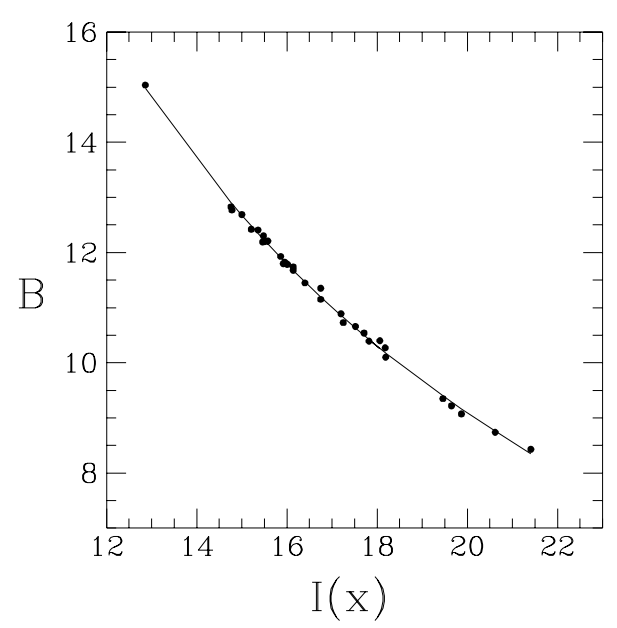

Fig. 1. Characteristic curve for the plate 19026. $I(x)$ are readings of the iris microphotometer "Sartorius" and $B$ are corresponding photoelectric stellar magnitudes from Table 5.

values of the internal rms errors of the photometry were adopted: $\sigma_{V}= \pm 0.04 \mathrm{mag}, \quad \sigma_{B}= \pm 0.06 \mathrm{mag}, \sigma_{B-V}=$ \pm 0.07 mag.

\section{Member segregation}

Proper motions used for open cluster membership segregation should be free of any systematic errors. The most important systematic error is the magnitude equation (ME) or the dependence of the observed star positions and the derived proper motions on stellar magnitudes.

If all observations were done with the same telescope and with a similar type of astroplate, under similar observational conditions (hour angles, zenith distances, coordinates of astroplate centers etc.) all star positions observed at different epochs would be contaminated by nearly the same systematic errors. The resulting proper motions, calculated as the difference of star positions at different epochs, would be free of the principal systematic errors.

The methods of ME estimation used at the Pulkovo Observatory were described in detail by Lavdovsky (1961). All proposed methods assume preliminary separation of cluster members from background stars and are based either on the analysis of proper motions of background stars only (first method), or of probable cluster members only (second method). 
In the first method, if we exclude from consideration the most dense part of the VPD, we exclude the main part of probable cluster members. At the same time, stars with very high proper motions ( $>50$ mas $\mathrm{yr}^{-1}$ ) should be eliminated too. The residual stars are (mostly) the background stars and their proper motions reflect the solar motion to the Apex. The background stars are distributed randomly in a space, so fainter stars are, on average, situated at larger distances from the Sun. Thus, the parallactic displacements caused by solar motion are smaller for fainter stars. These statistical parallactic displacements were calculated for different stellar magnitude ranges using the tables published by Zhukov (1966). When these statistical parallactic displacements are eliminated from the observed proper motions, the residual dependence on stellar magnitude presents the ME. The obvious disadvantage of this method is a very low precision of the secular parallaxes determined thus far. At best, this method permits us to estimate the linearity and the slope of ME for all observed stellar magnitudes.

In the second method, the estimation of the ME was based on the proper motions of cluster members preliminary selected by radial velocities. In Table 6 we collected all stars with known radial velocities in the cluster region. Data from HB and Barbier-Brossat \& Figon (2000) were used. Firstly, we excluded from consideration the stars known to be spectral binaries and determined the mean radial velocity $\left(\bar{V}_{\text {rad }}\right)$ and its standard deviation $(\sigma)$. After that, all stars with the radial velocities $\left|V_{\text {rad }}-\bar{V}_{\text {rad }}\right|>\sigma$ were excluded too. Thus, we obtained the mean cluster radial velocity of $-11.1 \pm 1.2 \mathrm{~km} \mathrm{~s}^{-1}$ for 22 stars marked with the symbol $\dagger$ in Table 6 . This value was accepted as the mean radial velocity of the cluster. If we consider that these 22 stars are real cluster members, they should have the same values of proper motions. But, in reality, the dependence of the observed proper motions on stellar magnitude was found for these 22 stars which permitted us to estimate the ME as $+0.59 \pm 0.07 \mathrm{mas} \mathrm{yr}^{-1} \mathrm{mag}^{-1}$ for $\mu_{x}$, and $0.00 \pm 0.05 \mathrm{mas} \mathrm{yr}^{-1} \mathrm{mag}^{-1}$ for $\mu_{y}$. However, because radial velocities were determined for a small number of bright stars ( $B \leq 12.5 \mathrm{mag}$ ) only, the value of ME remains unclear, as does the possibility of linear extrapolation of ME for faint stars.

To clarify the matter, another method of cluster member preselection was used. If we construct the CMD excluding stars with $B-V>1.0$ and lying far from the most dense part of the VPD, we can obtain the first approximation of the cluster main sequence. Using this criterion, we selected 310 probable cluster members. They allowed us to evaluate the ME as $+0.51 \pm 0.05 \mathrm{mas} \mathrm{yr}^{-1} \mathrm{mag}^{-1}$ for $\mu_{x}$, and $-0.35 \pm 0.04 \mathrm{mas} \mathrm{yr}^{-1} \mathrm{mag}^{-1}$ for $\mu_{y}$. These values confirm the character of the previous magnitude-restricted determination, but, and this is extremely important, they show the same character as the ME determined in the method of statistical parallaxes for all observed magnitudes $8.5 \mathrm{mag}<B<15.5 \mathrm{mag}$.

Finally, we tried to estimate the ME by comparing the obtained proper motions with absolute ones obtained in the space projects HIPPARCOS and Tycho-2. However, it is difficult to determine the ME comparing our proper motions with these of HIPPARCOS. Our catalogue of more than 2000 stellar proper motion determinations in the region of NGC 7243 covering a small field of 6400 square arcminutes contains only five stars in common with HIPPARCOS. All of them are in the 8.5-9.8 mag range and a comparison of the proper motions does not permit us to draw any significant conclusions about the ME.

The proper motions presented in the Tycho- 2 catalogue are derived mainly using photographically observed first epoch positions. However, a large number of stars in common with HIPPARCOS in all the observed magnitude range permitted us to correct the main part of the principal systematic errors, including ME, in Tycho-2. In the studied field of NGC 7243 we found 280 stars in common with Tycho-2. The ME obtained as the result of comparison of proper motions with Tycho-2 can be presented as:

$+0.52 \pm 0.17 \mathrm{mas} \mathrm{yr}^{-1} \mathrm{mag}^{-1}$ for $\mu_{x}$

$-0.53 \pm 0.22 \mathrm{mas} \mathrm{yr}^{-1} \mathrm{mag}^{-1}$ for $\mu_{y}$.

The low relative precision of the obtained ME and, in principal, the narrow range of stellar magnitudes does not permit one to apply them directly to correct the observed proper motions but permits us to confirm the previously determined values of ME.

On the other hand, the realized comparison permits one (taking into account the errors of our catalog) to estimate the accidental errors of Tycho-2 proper motions as:

$\sigma_{x}= \pm 3.0 \operatorname{mas~yr}^{-1}, \sigma_{y}= \pm 3.6 \operatorname{mas~yr}^{-1}$.

Finally, the ME obtained on the basis of 310 preliminary photometrically-selected cluster members was applied for every plate pair used for mean proper motion determinations. It was shown that the same ME, within the error bars, may be used for all plate pairs. This circumstance gave us the opportunity to use this ME in our following investigations applying the corrections caused by the ME to all determined proper motions.

The procedure for a final selection of cluster members described below permitted us to create a list of probable cluster members. The original (or observed) proper motions of these probable cluster members were studied to determine the existence of the ME. The ME obtained in the second approximation shows a linear character with the following regression coefficients (for the whole magnitude range):

$+0.49 \pm 0.05 \mathrm{mas} \mathrm{yr}^{-1} \mathrm{mag}^{-1}$ for $\mu_{x}$,

$-0.17 \pm 0.06 \mathrm{mas} \mathrm{yr}^{-1} \mathrm{mag}^{-1}$ for $\mu_{y}$.

All proper motions derived in our investigation were corrected using this ME for all observed stellar magnitudes. The resulting ME is shown in Fig. 2. The third approximation, carried out following the same scheme, does not show any significant residual ME.

The cluster member selection was done based on VPD and CMD. For these purposes, a preliminary catalogue was constructed including all stars having originally determined proper motions and reliably determined $B$ and $V$ magnitudes (in the published version of the catalogue the values without symbol “:”). In every possible case, photographic photometric data were substituted by their photoelectric counterparts. The VPD and CM diagram were constructed for all 1688 stars of this 
Table 6. Field stars and the cluster members selected in the previous works.

\begin{tabular}{|c|c|c|c|c|c|c|c|c|c|}
\hline \multirow[b]{2}{*}{$\begin{array}{l}\text { This } \\
\text { paper }\end{array}$} & \multicolumn{4}{|c|}{ Star numbers derived from } & \multicolumn{5}{|c|}{ Star numbers derived from } \\
\hline & Lengauer & Schewick & Muminov & $\begin{array}{c}V_{\mathrm{rad}} \\
\mathrm{km} \mathrm{s}^{-1} \\
\end{array}$ & $\begin{array}{l}\text { This } \\
\text { paper }\end{array}$ & Lengauer & Schewick & Muminov & $\begin{array}{c}V_{\mathrm{rad}} \\
\mathrm{km} \mathrm{s}^{-1}\end{array}$ \\
\hline 166 & 785 & & & +7.4 & 891 & $492 *$ & $59 *$ & 632 & \\
\hline 303 & 735 & & & -34.8 & $895^{\dagger}$ & 362 & $57 *$ & & -4.0 \\
\hline 350 & 754 & & & -27.8 & 896 & $361 *$ & $56^{*}$ & 625 & -11.0 \\
\hline $553^{\dagger}$ & 649 & & & -18.3 & 897 & $363^{*}$ & 58 & 626 & \\
\hline 616 & $500^{*}$ & 77 & 676 & & $902^{\dagger}$ & 358 & $49 *$ & 597 & -15.0 \\
\hline 743 & $476^{*}$ & 23 & 475 & -52.0 & 904 & $487 *$ & $52 *$ & 607 & -35.0 \\
\hline 744 & $473^{*}$ & $13^{*}$ & 440 & & 905 & 483 & $46^{*}$ & 586 & \\
\hline $757^{\dagger}$ & $482 *$ & $42 *$ & 567 & -8.0 & 909 & $480^{*}$ & $33 *$ & 516 & -11.0 \\
\hline $759^{\dagger}$ & $484^{*}$ & $44^{*}$ & 587 & -13.0 & $912^{\dagger}$ & $475^{*}$ & $21^{*}$ & $467 *$ & -13.0 \\
\hline $761^{\dagger}$ & $485^{*}$ & 48 & $592 *$ & -11.0 & 922 & $338^{*}$ & 7 & $393^{*}$ & \\
\hline 768 & 493 & & & +6.0 & $924^{\dagger}$ & $471^{*}$ & $11^{*}$ & 416 & -7.0 \\
\hline 771 & $490 *$ & $57 *$ & 628 & +5.0 & 1000 & 306 & & & +9.2 \\
\hline $775^{\dagger}$ & $497 *$ & 75 & 672 & -27.0 & 1068 & 364 & $65^{*}$ & 642 & \\
\hline $776^{\dagger}$ & $496^{*}$ & $71^{*}$ & 661 & -6.0 & $1075^{\dagger}$ & $368 *$ & $83^{*}$ & 696 & -5.0 \\
\hline 778 & $501 *$ & 78 & 682 & & $1078^{\dagger}$ & $370^{*}$ & $85^{*}$ & 714 & -12.0 \\
\hline $780^{\dagger}$ & $503 *$ & $80^{*}$ & 685 & -6.0 & 1081 & $374 *$ & 91 & 751 & \\
\hline $814^{\dagger}$ & 527 & & & -24.0 & 1084 & $378^{*}$ & $96^{*}$ & 769 & \\
\hline 869 & $382 *$ & 109 & 808 & & $1085^{\dagger}$ & $380 *$ & $102 *$ & 783 & -23.0 \\
\hline $872^{\dagger}$ & $512^{*}$ & $99^{*}$ & 785 & -18.0 & 1086 & 379 & $100 *$ & $782 *$ & -37.0 \\
\hline 876 & $510^{*}$ & $88^{*}$ & 731 & +3.0 & $1097^{\dagger}$ & $384 *$ & $111^{*}$ & 816 & -20.0 \\
\hline $879^{\dagger}$ & $377 *$ & $94 *$ & 765 & -13.0 & $1099^{\dagger}$ & 386 & 114 & 828 & -9.0 \\
\hline $881^{\dagger}$ & 372 & 89 & 745 & -4.0 & $1104^{\dagger}$ & 392 & 125 & 892 & -11.0 \\
\hline $886^{\dagger}$ & 369 & $84 *$ & 699 & +4.0 & $1737^{\dagger}$ & 181 & & & -14.3 \\
\hline
\end{tabular}

* - Previously determined cluster members.

$\dagger$ - Probable cluster members based on the radial velocity.

Stars 743, 768, 771, 896, 904, 909, 1085, and 1086 are spectroscopic binaries.
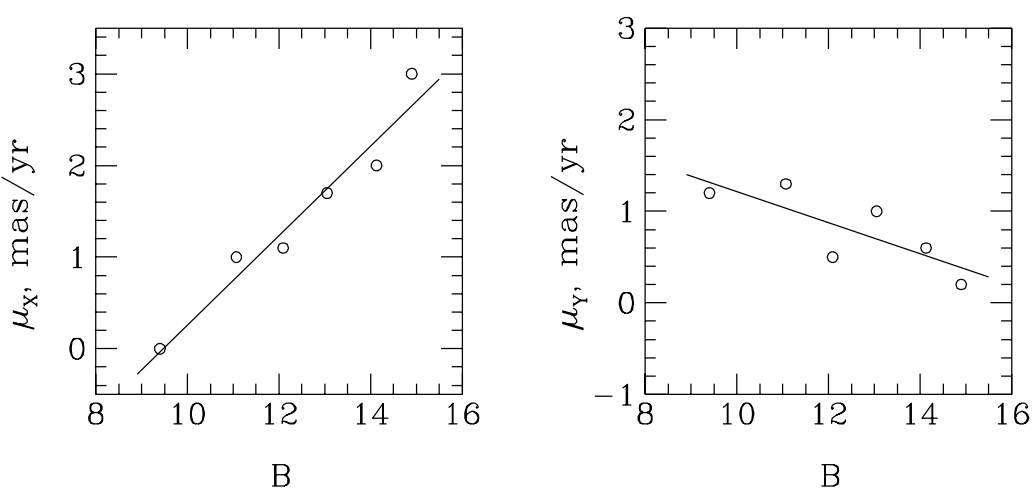

Fig. 2. Final magnitude equation. Points present mean proper motions of selected cluster members for different stellar magnitude groups. The analytical ME is obtained as a linear fitting to the $\mu_{x}$ and $\mu_{y}$ components.

preliminary catalogue (Figs. 3 and 4). Figure 5 presents an enlarged central part of the VPD. No condensation corresponding to the cluster can be seen.

Probable "astrometric" cluster members were determined by the formula proposed by Vasilevskis et al. (1958) and Sanders (1971) based on the idea that proper motion distributions for cluster and field stars are bivariate Gaussian distributions, circular for the cluster and elliptical for the field. Thus, each point of the VPD takes part in two Gaussian distributions: that of the cluster $\Phi_{\mathrm{c}}\left(\mu_{x}, \mu_{y}\right)$ and that of the field $\Phi_{\mathrm{f}}\left(\mu_{x}, \mu_{y}\right)$ :

$\Phi\left(\mu_{x_{i}}, \mu_{y_{i}}\right)=\Phi_{\mathrm{c}}\left(\mu_{x_{i}}, \mu_{y_{i}}\right)+\Phi_{\mathrm{f}}\left(\mu_{x_{i}}, \mu_{y_{i}}\right)$,

$$
\begin{aligned}
& \Phi_{\mathrm{c}}\left(\mu_{x_{i}}, \mu_{y_{i}}\right)= \\
& \frac{N_{\mathrm{c}}}{2 \pi \sigma^{2}} \times \exp \left\{-\frac{1}{2}\left[\left(\frac{\mu_{x_{i}}-\mu_{x_{0} \mathrm{c}}}{\sigma}\right)^{2}+\left(\frac{\mu_{y_{i}}-\mu_{y_{0} \mathrm{c}}}{\sigma}\right)^{2}\right]\right\}, \\
& \Phi_{\mathrm{f}}\left(\mu_{x_{i}}, \mu_{y_{i}}\right)=\frac{N_{\mathrm{f}}}{2 \pi \Sigma_{x} \Sigma_{y} \sqrt{1-r^{2}}} \\
& \times \exp \left\{-\frac{1}{2\left(1-r^{2}\right)}\left[\left(\frac{\mu_{x_{i}}-\mu_{x_{0} \mathrm{f}}}{\Sigma_{x}}\right)^{2}+\left(\frac{\mu_{y_{i}}-\mu_{y_{0} \mathrm{f}}}{\Sigma_{y}}\right)^{2}\right.\right. \\
& \left.\left.-2 r \frac{\left(\mu_{x_{i}}-\mu_{x_{0} \mathrm{f}}\right)}{\Sigma_{x}} \frac{\left(\mu_{y_{i}}-\mu_{y_{0} \mathrm{f}}\right)}{\Sigma_{y}}\right]\right\}
\end{aligned}
$$




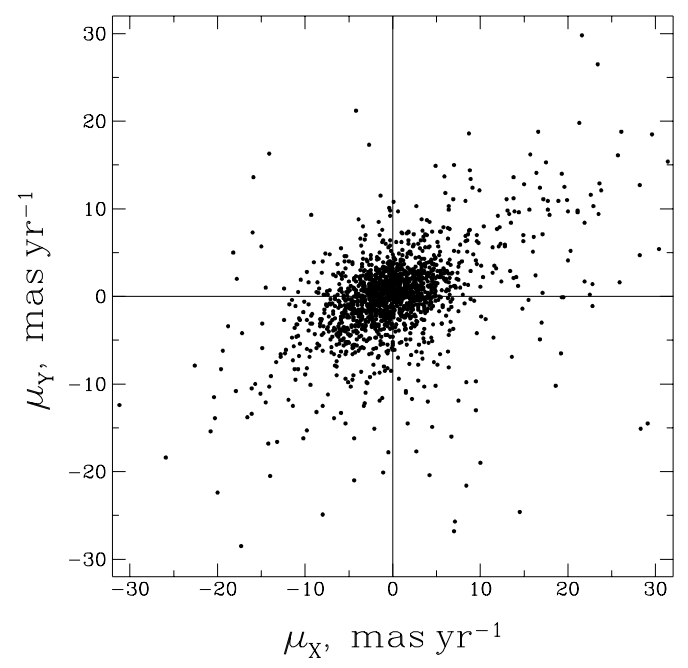

Fig. 3. The VPD for all stars in the region of the open cluster NGC 7243.

where $N_{\mathrm{c}}$ - normalized number of cluster stars,

$N_{\mathrm{f}}$ - normalized number of field stars,

$\sigma$ - standard deviations for stars of the cluster,

$\Sigma_{x}, \Sigma_{y}$ - standard deviations for the field stars,

$r$ - correlation coefficient, relating to the orientation of the ellipse describing the distribution of field stars,

$\mu_{x_{0} \mathrm{c}}, \mu_{y_{0} \mathrm{c}}, \mu_{x_{0} \mathrm{f}}, \mu_{y_{0} \mathrm{f}}-$ coordinates of centers of distribution on the VPD of the cluster and field stars,

$\mu_{x_{i}}, \mu_{y_{i}}$ - proper motion in $x$ and $y$ for the $i$ th star.

Individual membership probability of a star is determined by its position on the VPD and is calculated by the following formula:

$P\left(\mu_{x}, \mu_{y}\right)=\frac{N_{\mathrm{c}} \Phi_{\mathrm{c}}\left(\mu_{x}, \mu_{y}\right)}{N_{\mathrm{c}} \Phi_{\mathrm{c}}\left(\mu_{x}, \mu_{y}\right)+N_{\mathrm{f}} \Phi_{\mathrm{f}}\left(\mu_{x}, \mu_{y}\right)}$.

For calculating the membership probabilities it is necessary to determine the parameters of each distribution. For this purpose, Sanders (1971) suggested using a method of maximum likelihood. Thus, it is necessary to solve a system of nonlinear equations, the number of which corresponds to the number of unknown parameters.

In our study, the Sanders' method cannot be used directly to fit all 8 unknown parameters of the Gaussian proper motion distributions due to a rather small ratio of cluster and field stars. The cluster NGC 7243 region of $80^{\prime} \times 80^{\prime}$ contains more than 2600 stars up to $B=15.5$ mag while according to previous investigations the cluster diameter is about 21' (Lyngå 1987) and the number of cluster members determined by Lengauer (1933) is only 32 . That is why it was necessary to subject the VPD to preliminary pruning. The frequency distribution parameters for field and cluster stars were chosen iteratively. At the beginning, all originally-determined proper motions were transformed to the new coordinate system $\left(\mu_{x^{\prime}}, \mu_{y^{\prime}}\right)$ by rotating the initial coordinate system so that the large axis of the ellipse coincides with the $x$ (Right Ascension) axis. The preliminary center of the cluster proper motion distribution was determined in this new coordinate frame. For a preliminary cluster member

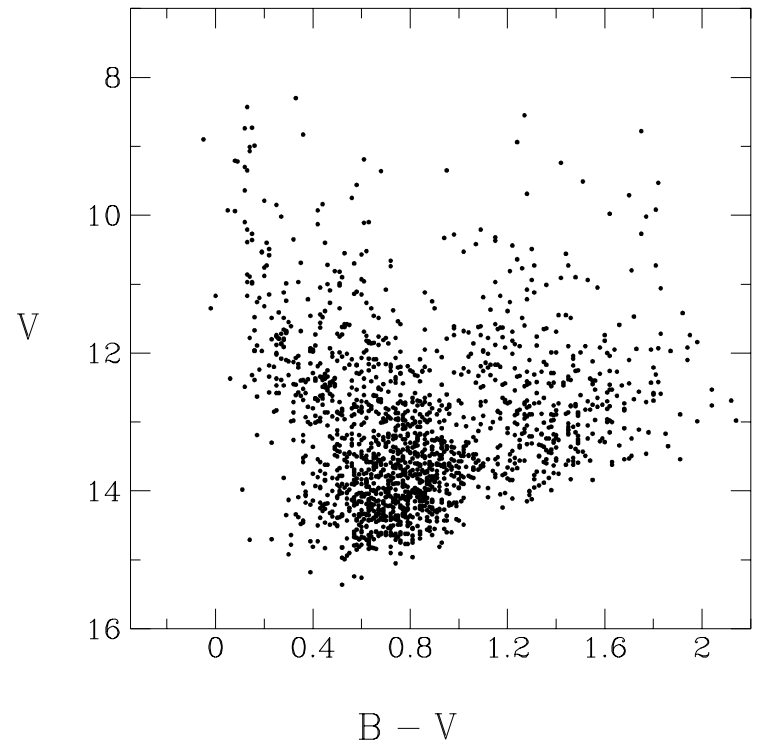

Fig. 4. CM diagram for all stars in the region of the open cluster NGC 7243.

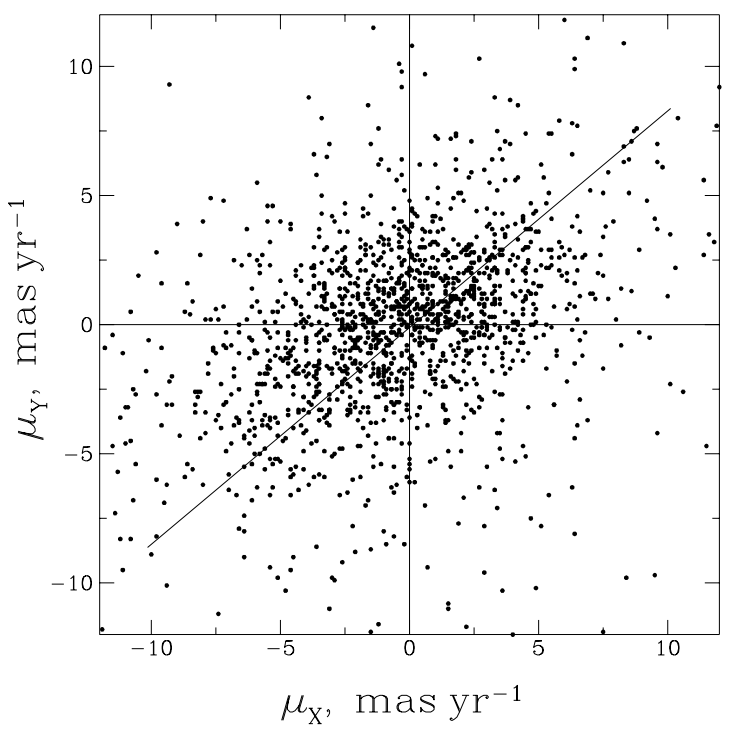

Fig. 5. The central part of the VPD with the orientation of the large axis of the elliptic proper motions distribution.

separation, a procedure similar to the case of ME determination was used.

The visual analysis of the CMD shows that the stars with $B-V>1.0$ are improbable cluster members and might be excluded from consideration. So, the elimination of these stars will clean the central part of the VPD. On the other hand, stars with large proper motions $\left(\left|\mu_{x^{\prime}}, \mu_{y^{\prime}}\right|>50\right.$ mas $\left.\mathrm{yr}^{-1}\right)$ might be eliminated from consideration too.

Taking into account that the cluster diameter is 21' (Lyngå 1987), stars lying at distances more than $25^{\prime}$ from the geometrical center of the cluster were eliminated.

We tried to find the center of the cluster proper motion distribution as the region of the highest density on cleaned VPD. For this purpose, we compared the proper motions of each star, $\mu_{x_{i}^{\prime}}$ and $\mu_{y_{i}^{\prime}}$, with the proper motions of all residual stars and 


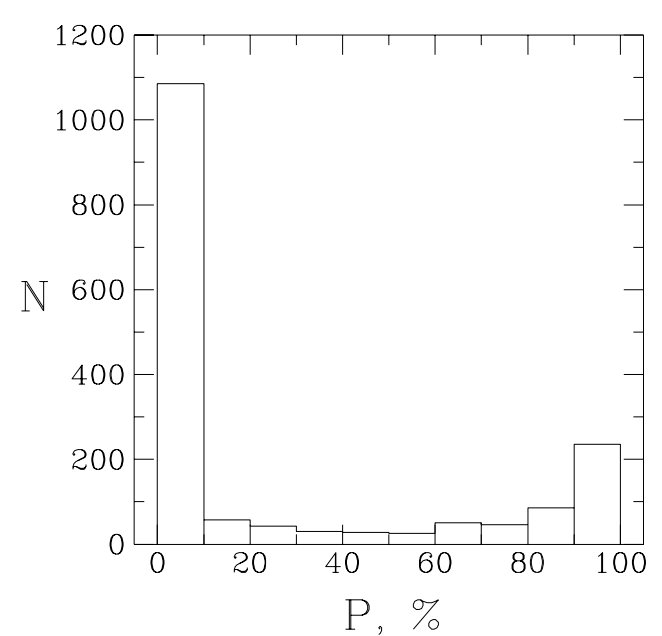

Fig. 6. Membership probability histogram for all stars in the cluster region.

counted the number of stars $N_{i}$ lying within the circle centered on $\mu_{x_{i}^{\prime}}$ and $\mu_{y_{i}^{\prime}}$ with the radius determined by errors of

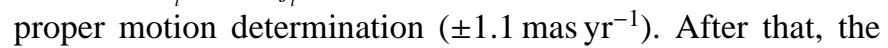
point with the coordinates $\left(\mu_{x_{i}^{\prime}}, \mu_{y_{i}^{\prime}}\right)$ was assigned the weight $W_{i}=N_{i}$. When this comparison was made for all stars of the cleaned VPD we found the mean weight $W_{0}$. At the second step, all points on the VPD with $W_{i}<W_{0}$ were eliminated as corresponding to improbable cluster members. For all stars with $W_{i}>W_{0}$ the position of cluster center on the VPD was calculated as a barycenter:

$\mu_{x_{0}^{\prime}}=\frac{\Sigma \mu_{x_{i}^{\prime}} \cdot W_{i}}{\Sigma W_{i}}, \quad \mu_{y_{0}^{\prime}}=\frac{\Sigma \mu_{y_{i}^{\prime}} \cdot W_{i}}{\Sigma W_{i}}$.

It is impossible to determine the center of the very noisy proper motion distribution of field stars using only VPD, so it was assumed that $\mu_{x^{\prime}}=0$ and $\mu_{y^{\prime}}=0$. The ellipse semi-axes or standard deviation of the field stars proper motion distribution were determined by VPD. The numbers of the cluster members $N_{\mathrm{c}}$ and field stars $N_{\mathrm{f}}$ as well as their ratio $N_{\mathrm{c}} / N_{\mathrm{f}}=1.03$ were determined from this first approximation. Using these values of the distribution parameters, individual membership probabilities $(P)$ were calculated.

At the second approximation, only stars with $P>80 \%$ were considered as astrometric cluster members. Figure 6 shows the histogram of the membership probability.

Then, all stars with membership probabilities $P>80 \%$ were excluded from previously selected field stars, and afterwards new values of the distribution parameters of field stars were obtained. In this case, Sanders' method was used independently to fit 5 unknowns using only $\mu_{x^{\prime}}=0$ and $\mu_{y^{\prime}}=0$ as the first approximation of parameters obtained from the first it-

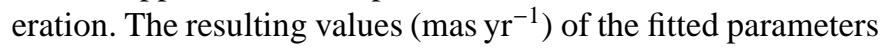
of the field and the cluster star distributions are the following:

\begin{tabular}{|c|c|c|c|c|c|}
\hline The field & $\mu_{x_{0}^{\prime}}=$ & -1.5 & The cluster & $\mu_{x_{0}^{\prime}}=$ & 2.1 \\
\hline & $\mu_{y_{0}^{\prime}}=$ & 0.5 & & $\mu_{y_{0}^{\prime}}=$ & -1.3 \\
\hline & $\Sigma_{x^{\prime}}=$ & 9.0 & & $\sigma_{x^{\prime}, y^{\prime}}=$ & 1.1 \\
\hline & $\Sigma_{y^{\prime}}=$ & 6.5 & & & \\
\hline
\end{tabular}

The fitted distributions are shown in Fig. 7 where they can be compared with histograms of the observed proper motion distributions.

The star position on the cluster CM diagram was accepted as a photometric criterion of the cluster membership. The shape of the diagram (see Fig. 8) clearly reflects the fact that $\sigma_{B-V}$ of $B-V$ determination increases with stellar magnitude. All stars with astrometric membership probabilities more than $80 \%$ and lying in a band of $\pm 3 \sigma_{B-V}$ were considered to be cluster members. The total number of such stars is 209 . Two bright stars, 895 and 896, were added to our list of cluster members according to the results of previous determinations of their radial velocities and photoelectric $U B V$ magnitudes. Unfortunately, these stars form one undistinguished image on our plates. So the total number of stars which are cluster members is 211 .

The comparison of the cluster and the field magnitude functions (Fig. 9) shows that the obtained number of cluster members is restricted by the depth of the astrometric plates. From Fig. 10 presenting the $X Y$ positions of all the stars in the region of NGC 7243, we can see that the field stars are homogeneously distributed over the whole area.

\section{CM diagram and cluster physical parameters}

\section{a) Reddening and distance}

The selective absorption in the region of the cluster was determined using 34 cluster members that satisfied the following conditions: 1) existence of the $U B V$ photoelectric magnitudes, and 2) luminosity classes IV and V. The best coincidence was achieved by overlapping the normal colour line and the position of stars on the colour-colour diagram at the value of $E_{B-V}=0.24 \mathrm{mag}$. The standard inclination of a reddening line, $E_{U-B} / E_{B-V}=0.72$, was taken. The results are shown in Fig. 11. The value of colour excess for $B-V$ completely coincides with similar determinations in the works of MD and HB. The last authors applied also the data of the intermediateband $u v b y H \beta$ photometry and showed that the absorption in the region of the cluster core can be considered as uniform. HB, based on their spectral MK classification, obtained a slightly different value of reddening: $E_{B-V}=0.19 \pm 0.05 \mathrm{mag}$.

The visible distance modulus was obtained by a shift of ZAMS on the CM diagram along the axis $V$ at $E_{B-V}=$ 0.24 mag up to its coincidence with the MS of the cluster. Despite a noticeable scattering of the points, the distance modulus can be determined due to the fact that the positions of ten stars, which are highly probable cluster members (based on their astrometric, photometric and radial velocities determinations), form a visible thickening on the diagram shown by a circle in Fig. 12, where the final CM diagram of 211 members of the open cluster NGC 7243 is plotted. Its middle point is $V=11.74 \mathrm{mag}, B-V=0.28 \mathrm{mag}$, and the corresponding value of the visible distance modulus is $V-M_{V}=9.94 \mathrm{mag}$, that at a general absorption of $A_{V}=0.72$ mag results in the true modulus of $V_{0}-M_{V}=9.22 \mathrm{mag}$. The points of the lines of normal colours and ZAMS were taken from the work of Mermilliod (1981) where they were determined using open clusters younger than Hyades. 

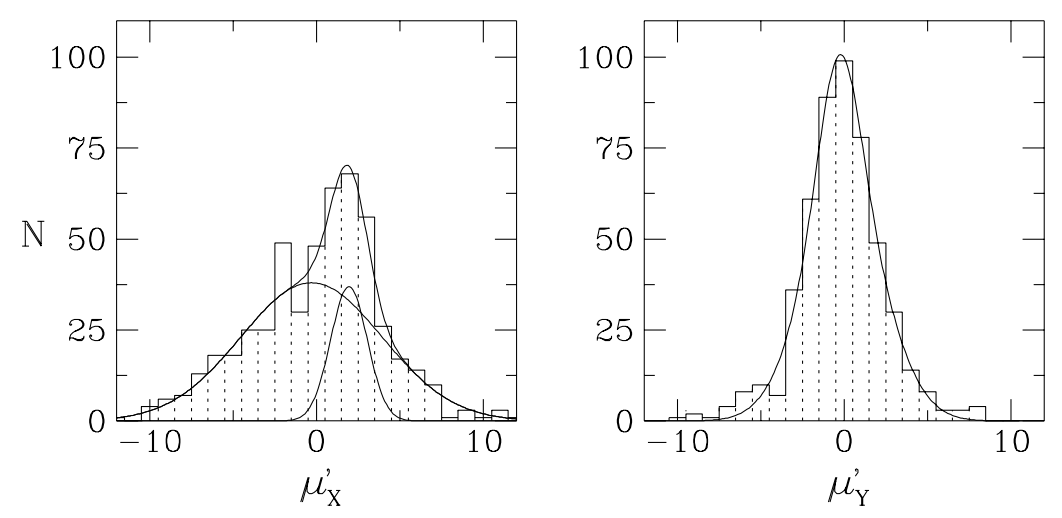

Fig. 7. Histograms of the proper motions $\mu_{x^{\prime}}$ and $\mu_{y^{\prime}}$ distributions and their approximations by fitted Gaussian distributions for cluster and field stars.

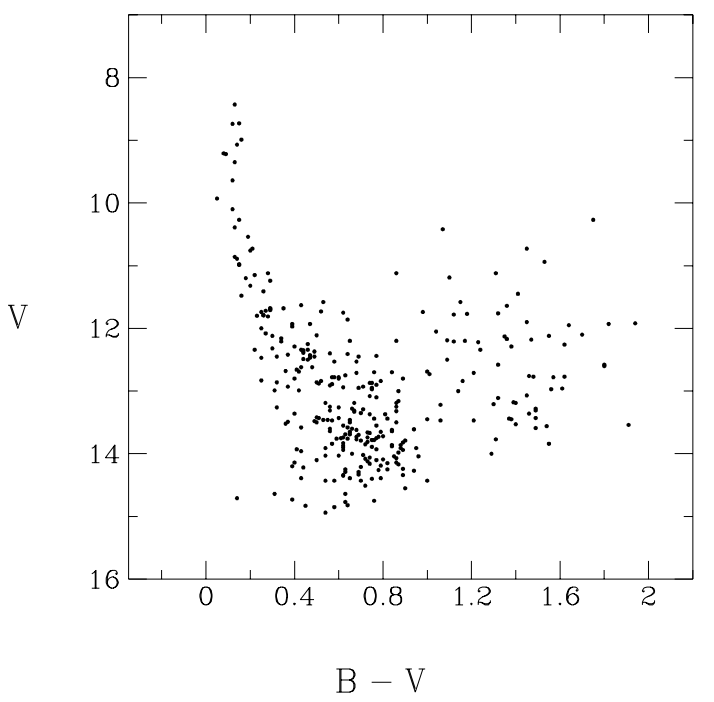

Fig. 8. CM diagram for probable cluster members with astrometric membership probabilities $P>80 \%$.

The determination of the average values of the visible modulus for astrometric members by MK classification results in the value of $V-M_{V}=9.94 \pm 0.37 \mathrm{mag}$ that corresponds to a distance of $r=698_{-109}^{+130} \mathrm{pc}$.

\section{b) Cluster age}

The age of the cluster was estimated based on the $(B-V)_{0}$ value of the turn-off point. This value for NGC 7243 is $(B-$ $V)_{0}=-0.11 \mathrm{mag}$. For such data the cluster age was estimated as $(2.5 \pm 0.5) \times 10^{8} \mathrm{yr}$ by the use of the isochrone grids computed by the Padova theoretical group (Bertelli et al. 1990). The main sequence and three isochrones corresponding to the ages of $1 \times$ $10^{8}, 2 \times 10^{8}$, and $3 \times 10^{8}$ yrs are shown in Fig. 13 .

\section{c) Luminosity and mass functions. Cluster mass estimation}

The cluster luminosity function, $\phi\left(M_{V}\right)$, is determined by the following equation: $\mathrm{d} N=\phi\left(M_{V}\right) \mathrm{d} M$, where $\mathrm{d} N$ - number of stars with absolute magnitudes in the limits $M_{V}, M_{V}+\mathrm{d} M_{V}$.

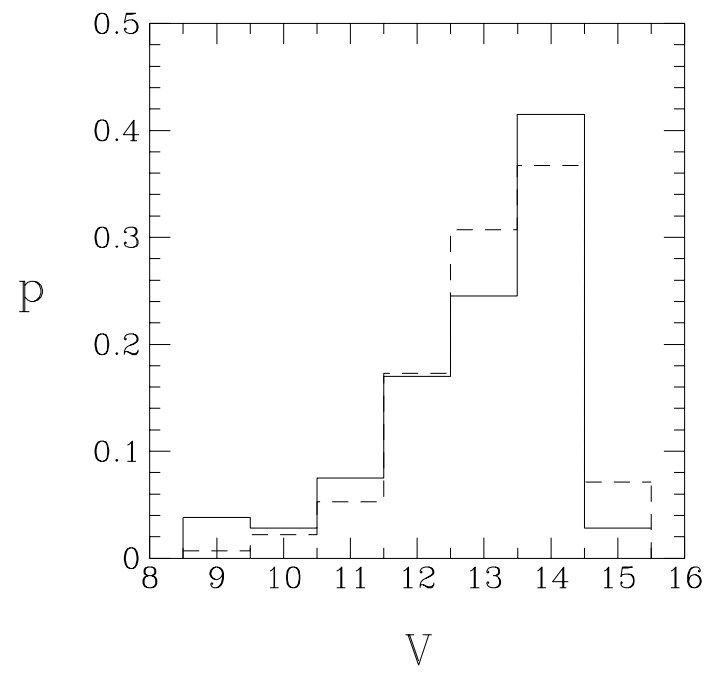

Fig. 9. The cluster (full line) and the field (dash line) magnitude functions.

The normalized luminosity function for NGC 7243 based on our data is given in Fig. 14.

Star masses were derived from the "mass-luminosity" relations published by Straizys \& Kuriliene (1981). Then, the empirical mass function for NGC 7243 was constructed. Salpeter (1955) was the first to show that for the stars in the vicinity of the Sun the power law of the initial mass function (IMF) has the form of a power law $\mathrm{d} N / \mathrm{d} M=\left(M / M_{\odot}\right)^{\alpha}$, with $\alpha=-2.35$. Numerous recent investigations of star clusters and associations of the Milky Way and the LMC exhibit a large range of logarithmic slopes in any mass range. Scalo (1998), assembling and analyzing these data, proposed a three-segment power law IMF:

$\mathrm{d} N / \mathrm{d} M=\left\{\begin{array}{l}-1.2 \pm 0.3 \text { for } 0.1<M / M_{\odot}<1 \\ -2.7 \pm 0.5 \text { for } 1<M / M_{\odot}<10 \\ -2.3 \pm 0.5 \text { for } 10<M / M_{\odot}<100 .\end{array}\right.$

In our investigation the logarithmic mass function (Fig. 15) was obtained as rms fitting for stars in the mass range $1<M / M_{\odot}<$ 6.5 in the form

$\lg N=-2.37 \lg \left(M / M_{\odot}\right)+2.18$ 
a)

b)

c)

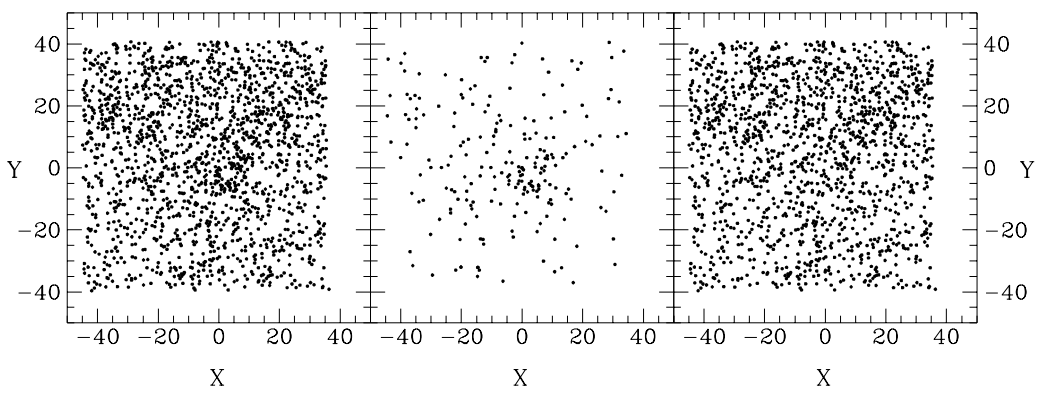

Fig. 10. The position diagram for the NGC 7243 region: $a$ ) all stars in the cluster region, $b$ ) selected cluster members, $c$ ) the field stars only.

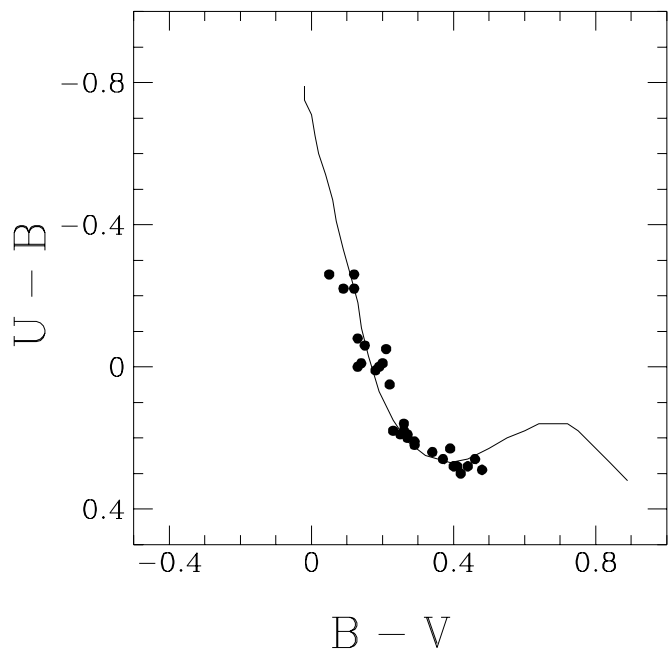

Fig. 11. The two colour diagram of the open cluster NGC 7243 approximated with a line of normal colours.

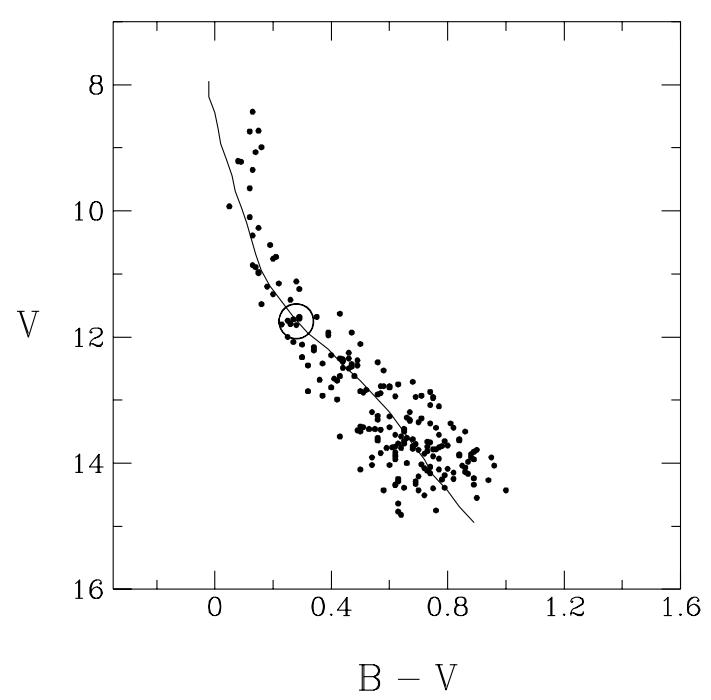

Fig. 12. CM diagram for the open cluster NGC 7243 members. The circle marks the positions of ten stars, which are reliable cluster members. ZAMS is shown by a solid line.

Thus the inclination of the mass function of NGC7243 $(-2.37 \pm 0.27)$ is in good agreement with $\alpha=-2.3$ recently obtained by Meusinger et al. (1996) for stars with $1.1<M / M_{\odot}<$ 3.5 of the Pleiades cluster.

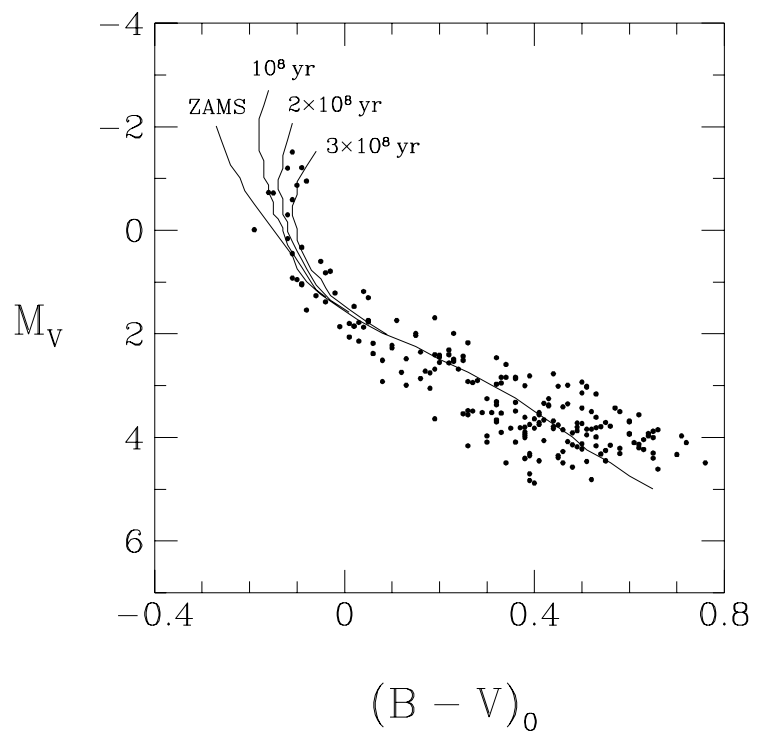

Fig. 13. The CM diagram with ZAMS and three isochrones corresponding to different cluster ages.

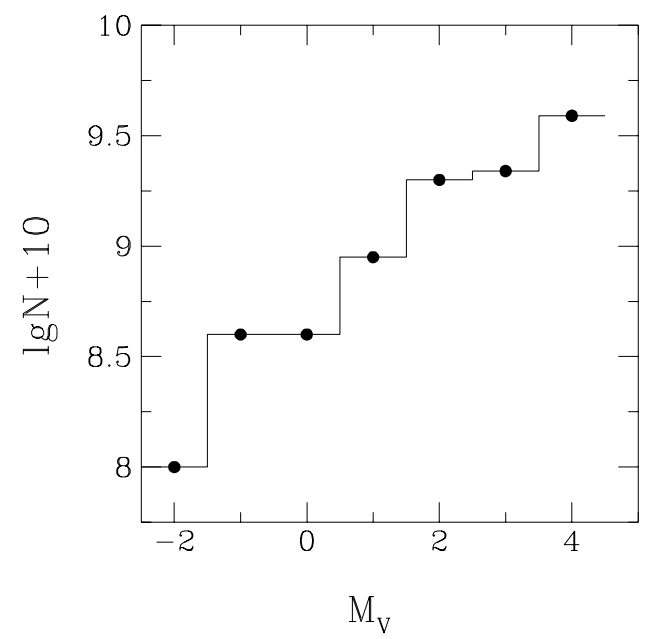

Fig. 14. The normalized luminosity function for the open cluster NGC 7243.

The estimation of the cluster mass was made on the following assumptions. The direct determinations of masses of each of its members based on the mass-luminosity correlation give a total value of $348 M_{\odot}$. Assuming that half of them are double stars with a ratio of masses of components close to unity, we 


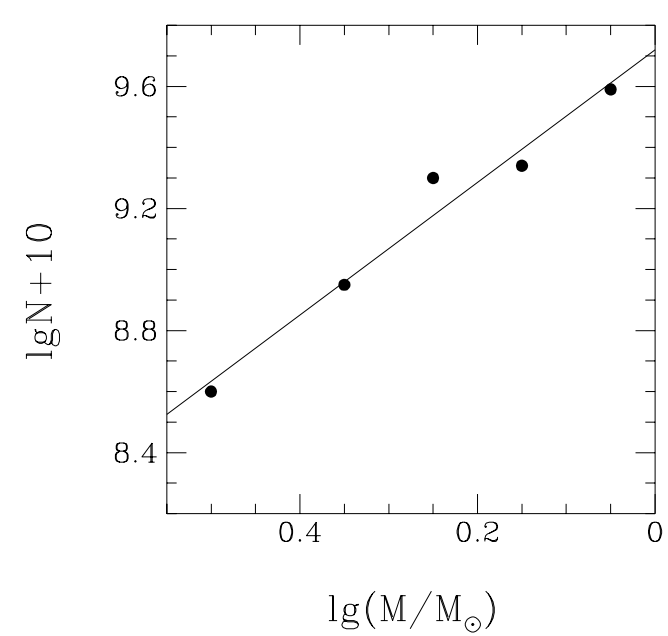

Fig. 15. Mass function of the open cluster NGC 7243.

should increase this value 1.5 times. The resulting estimation of $522 M_{\odot}$ is still not final since it is possible that a part of the faint cluster members has remained unrevealed because of the depth limit of the astrometric plates.

\section{d) Variable and peculiar stars in the region of the cluster}

According to the General Catalogue of Variable Stars (1985) and the New Catalogue of Suspected Variable Stars (1982) there are 8 known and 3 suspected variable stars in the studied region. Four of them are identified in our catalogue (see Table 8, column of notes) but none of them are cluster members. Among the suspected variables two are not members and the third - number 909 in our catalogue (number 480 in the Lengauer one) - is one of the brightest stars of the NGC 7243, a spectral binary with attributes of weak brightness fluctuations according to HB observations. Our photometry does not allow us to prove or disprove this fact, so the issue remains open. On the other hand, three other stars from our catalogue (863, 1634 and 2278) have shown significant divergences in $B$ and $V$ magnitudes obtained with the different plates of the Schmidt telescope. The star 863 has a high membership probability by proper motions $-P=97 \%$. According to this criterion, among the cluster members there are also two stars with unusual spectra: number $904-$ Am with $P=85 \%$ and $1078-$ A0 Vp with $P=96 \%$.

\section{Conclusions}

We have carried out an astrometric and photometric study of the young open cluster NGC 7243, poorly investigated before. The main cluster parameters obtained in this investigation are given in Table 7. The complete catalogue of 2623 stars in the region of the NGC 7243 containing positions, proper motions and their individual errors, $B$ and $B-V$ magnitudes and individual membership probabilities (Table 8 ) is available in electronic form at the CDS.
Table 7. NGC 7243 summary data.

\begin{tabular}{ll}
\hline \hline Number of members & 211 \\
Limiting magnitude & $V \sim 15.5 \mathrm{mag}$ \\
Magnitude of the brightest star & $V=8.43 \mathrm{mag}$ \\
Earliest spectral type & $\mathrm{B} 5 \mathrm{III}$ \\
MS turn-off point & $(B-V)_{0}=-0.11 \mathrm{mag}$ \\
Age & $t=(2.5 \pm 0.5) \times 10^{8} \mathrm{yr}$ \\
Total cluster mass & $348 M_{\odot} \leq M_{\mathrm{TOT}} \leq 522 M_{\odot}$ \\
Average reddening & $E_{B-V}=0.24 \mathrm{mag}$ \\
Distance & $r=698_{-109}^{+130} \mathrm{pc}$ \\
Mass function slope & $-2.37 \pm 0.27$ \\
\hline
\end{tabular}

Acknowledgements. E.G.J. thanks CNPq and FAPERJ for financial support under contracts 300016/93-0 and E-26/152.221/2000. N.A.D. thanks FAPERJ for financial support under contracts E-26/151.172/98 and E-26/171.647/99.

\section{References}

Barbier-Brossat, M., \& Figon, P. 2000, A\&AS, 142, 217

Bertelli, G., Betto, R., Bressan, A., et al. 1990, A\&AS, 85, 845

Cabrera-Caño, J., \& Alfaro, E. J. 1985, A\&A, 150, 298

General Catalogue of Variable Stars, Vol. II, 1985, ed. P. N. Kholopov (Moskow: Nauka)

Grave De 1979, Vatican Obs. Publ. 1, No 16, 283

Hill, G., \& Barnes, J. V. 1971, AJ, 76, 110

King, D. S. 1979, Sydney Obs. Papers, No 85

Kuznetsov, V. I., Lazorenko, G. A., \& Lazorenko, P. F. 1993, A\&A, 278,43

Lasker, B. M., Sturch, C. R., Lopez, C., et al. 1988, ApJS, 68, 1

Lavdovsky, V. V. 1961, Tr. Glav. Astron. Obs. Pulkovo, Ser., 2, 73, 5

Lengauer, G. G. 1937, Izv. Glav. Astr. Obs. Pulkovo, XV, 126, 42

Lyngå, G. 1987, Catalogue of Open Cluster Data, 5th edition

Mermilliod, J. C. 1981, A\&A, 97, 235

Meusinger, H., Schilbach, E., \& Souchay, J. 1996, A\&A, 312, 833

Mianes, P., \& Daguillon, J. 1956, Publ. L'Obs. Haute-Provence, 3, No 57, 65

Missana, M., \& Missana, N. 1990, AJ, 100, 1850

New Catalogue of Suspected Variable Stars, 1982, ed. P. N. Kholopov (Moskow: Nauka)

Raab, S. 1922, Lund Meddelande, Ser. 2, 30, 64

Rahmatov, E., \& Muminov, M. 1985, Tashkent Astr. Inst. Circ., 116, 17

Salpeter, E. 1955, ApJ, 121, 161

Sanders, W. L. 1971, A\&A, 14, 226

Scalo, J. M. 1998, ASP Conf. Ser., 142, 201

Slovak, M. N. 1977, AJ, 82, 818

Straižys, V., \& Kuriliene, G. 1981, Ap\&SS, 80, 353

Trümpler, R. J. 1930, Lick Obs. Bull., 14, 154

van Schewick, H. 1957, Veröf. Univ.-Sternwarte Bonn, 47, 1

Vasilevskis, S., Klemola, A., \& Preston, G. 1958, AJ, 69, 387

Weaver, H. 1962, Handbuch der Physik, 54, 130

Zhao, J. L., \& He, Y. P. 1990, A\&A, 237, 54

Zhukov, L. V. 1966, AZh, 43, 1107

Zug, R. S. 1933, Lick Obs. Bull., 16, 135 